\title{
Article
}

\section{On the Use of Inverse Methods to Parameter Estimation in Turbulent Pipe Flows of Drag Reducing Polymers}

\author{
Mohammad Allahdadi-MeHrabadi, Kayvan SADEGHY ${ }^{\dagger}$, Ali HaKKAKI-FARD, and Mostafa Sefidgar \\ University of Tehran, College of Engineering, Department of Mechanical Engineering \\ P.O. Box: 11155-4563, Tehran, Iran \\ (Received : May 7, 2008)
}

\begin{abstract}
This article investigates the applicability of inverse methods in estimating the best values to be assigned to certain parameters which appear in turbulent flow studies of dilute polymer solutions in circular pipes. These parameters naturally arise when the Nagano-Hishida low-Re, $k-\varepsilon$ model is combined with a special form of the Generalized Newtonian Fluid model modified in such a way that it could account for the elasticity of dilute polymer solutions. This multi-objective problem is treated by a positively weighted convex sum of the objectives. Then the procedure is followed by a well-known Gauss-Newton nonlinear optimization method for optimizing model parameters in order to better predict the drag reduction phenomenon using polymeric additives. Parameter optimization relies on the availability of experimental data for the velocity profiles, friction factor, and turbulence kinetic energy for at least one concentration of a given polymer. It is shown that using these optimized parameters, it is possible to predict with more accuracy the amount of drag reduction achievable using a given polymeric additive. It is also shown that these parameters may neither work for other concentrations of the same polymer nor necessarily for any concentration of any other polymer, inferring that for non-Newtonian fluids these parameters are not at all universal.
\end{abstract}

Key Words: Turbulence modeling / Drag Reduction / Parameter Optimization / Inverse Method / Polymeric Additives

\section{INTRODUCTION}

In recent years, inverse methods have evolved as an efficient tool to tackle with virtually all sorts of engineering problems. ${ }^{1)}$ The heat transfer community, for example, has found such methods very useful for estimating surface heat flux provided temperature history within a conducting body. ${ }^{2)}$ Inverse methods have been used with great success to estimate other unknowns such as: i) missing initial or boundary conditions, ii) missing physical/rheological properties of a material, and iii) missing geometrical parameters involved in a given problem. ${ }^{2,3)} \mathrm{A}$ number of inverse methods have been developed over the years to tackle with such engineering problems. One can mention, for example, the sequential function-specification method ${ }^{2}$, the regularization method $^{4}$, the iterative regularization method ${ }^{5)}$, the Levenberg-Marquardt method $^{6,7)}$, the conjugate gradient method ${ }^{8}$, the variable metric method $^{9)}$, and the genetic algorithm method. ${ }^{10-12)}$ In virtually all of these inverse methods, the optimization process involves minimization of an objective function which includes both the

$\uparrow$ To whom correspondence should be addressed.

Phone: +9821-6111-4011, Fax: +9821-8801-3029, E-mail: sadeghy@ut.ac.ir computed values and the measured values.

A potential field of application for inverse methods appears to lie in studies related to turbulent flows. That is, turbulent flows by their very nature involve fluctuations in all sorts of physical quantities (say, velocity, temperature, pressure, heat flux, etc). And because experimental data are always accompanied with some degree of uncertainty, therefore an optimization scheme appears to be most desired in such turbulent flow studies. To this should be added the fact that, the field of turbulence modeling also involves assigning numerical values to certain "parameters" inherent in such models. These parameters are empirical in nature and their determination depends on the availability of a sufficient amount of experimental data. Evidently, the field of turbulent flow appears to be well suited to one of the available inverse methods. As a matter of fact, Liu and Ozisik ${ }^{13)}$ made use of the conjugategradient method with an adjoint equation to estimate heat flux fluctuations in turbulent duct flow. $\mathrm{Li}$ and $\mathrm{Yan}^{14)}$ relied on a similar inverse method for estimating heat flux fluctuations in forced-convection turbulent flow between two parallel plates. Su et al. ${ }^{15)}$ applied the Levenberg-Marquardt inverse method to estimate non-uniform heat flux for a thermally-developing turbulent pipe flow using temperature measurement at several 
locations along the pipe. Su and Silva Neto ${ }^{16)}$ used an inverse method for simultaneous estimation of the inlet temperature profile and also the wall heat flux distribution in turbulent pipe flow. Brasil and $\mathrm{Su}^{17)}$ relied on an inverse method for estimating the upstream velocity profiles in an incompressible turbulent boundary layer. Storcha and Pimentela ${ }^{18)}$ made use of an inverse method to identify atmospheric boundary layer parameters. King and Bloor ${ }^{19)}$ invoked a semi-inverse method for free-surface flow over a submerged body.

The inverse turbulent-flow studies mentioned above were all concerned with Newtonian fluids. Quite surprisingly, however, there appears to be no inverse analysis whatsoever in relation to turbulent flows of non-Newtonian fluids. This is rather surprising realizing the fact that turbulent flows of non-Newtonian fluids has emerged as an equally-important field of study since Toms' discovery that it is possible to reduce friction drag in turbulent pipe flows using certain polymeric additives. ${ }^{20)}$ Indeed, since Toms' experimental finding, much study has been done in the theoretical domain to better understand this phenomenon. Of prime importance in such studies has been to predict the remarkable drag reduction which is attainable in practice by a few ppm of certain polymeric additives. The level of the drag reduction which may be obtained using this method is quite striking as it may amount up to $80 \%$ (depending on the polymer used). Quite surprisingly, however, previous studies have failed to predict such remarkable reduction in the drag. In fact, even the powerful direct numerical simulation (DNS) performed on the basis of well-known versatile viscoelastic fluid models such as the Giesekus and FENE models have not been so successful in predicting this much reduction in the turbulent viscous drag. This is quite disappointing realizing the fact that direct numerical simulation, albeit admittedly very expensive and time-consuming, renders highly accurate results for Newtonian fluids.

In an attempt to develop a practical, more affordable method to predict the level of the drag reduction achievable using polymeric additives, Pinho ${ }^{21)}$ developed a time-averaged turbulent-flow formulation based on one of the simplest constitutive equations available for non-Newtonian fluids, i.e., the Generalized Newtonian Fluid (GNF) model. In practice, however, Pinho ${ }^{21)}$ had to modify this rheological model in such a way that it could account for both shear-thinning and strainthickening behavior of dilute polymer solutions (as required by any realistic simulation of dilute polymer solutions). Having integrated this particular viscoelastic fluid model with the Nagano-Hishida $k-\varepsilon$ turbulence model, Cruz and Pinho ${ }^{22)}$, and Cruz et $a l .{ }^{23)}$ managed to predict, for the first time, the level of the huge drag reduction which is observed in turbulent pipe flows of dilute polymer solutions. Their prediction for the friction factor is in good quantitative agreement with experimental data, but, there certain polymer solutions for which the agreement appears not to be so great. ${ }^{22,23)}$

The rather poor agreement between numerical results and experimental data for certain polymer solutions ${ }^{22,23)}$ may have simply arisen because the fluid under investigation does not perfectly obey the modified GNF rheological model as proposed by Pinho.. ${ }^{21)}$ Another explanation for the abovementioned discrepancy may lie in the fact that the NaganoHishida $k-\varepsilon$ model may not necessarily be the most appropriate turbulence model for all polymer solutions. As a matter of fact, in a recent work ${ }^{24)}$ it has been shown that by relying on another $k-\varepsilon$ turbulence model, i.e., the Launder-Sharma model, better agreement can be obtained between simulations and experimental data for certain polymer solutions. Another scenario which can explain the rather poor performance of Nagano-Hishida $k-\varepsilon$ method for certain polymer solutions appears to be the numerical values assigned to the empirical parameters inherent in any turbulence modeling. That is, for certain parameters in this turbulence model use has been made of Newtonian values, and for certain other parameters a trialand-error method has been invoked based on data available for just a single polymer solution. Thus, it should not be too surprising to see that the parameters so assigned are not so universal, as originally thought.

In the present study, it is shown that the values assigned to these parameters (to be introduced shortly) all have a profound effect on the quality of the numerical results in drag reduction studies of dilute polymer solutions. That is, we show that the quality of numerical predictions can be improved if these “parameters” are refined appropriately for any given polymer solution. The method of refinement adopted in the present work is one of the inverse methods. That is, we are going to resort to a suitable optimization method to determine the best values for these parameters for each polymeric additive so that numerical predictions for friction drag can better match experimental data, at least for the same polymer at any other concentration. To the best of our knowledge, this is the first treatment of turbulent flows of non-Newtonian fluids within an inverse context.

\section{THE DIRECT PROBLEM}

An important step in any theoretical analysis of drag reduction phenomenon in turbulent pipe flows of polymer solutions is to decide on the constitutive behavior of the fluid. 
In the present work, we are going to rely on the modified Generalized Newtonian Fluid model as proposed recently by Pinho $^{21)}$ for this purpose. In this modified version of the GNF, the viscosity coefficient is written in such a way that it becomes a function of both shear rate and strain rate; that is:

$$
\mu=K_{v}[\dot{\gamma}]^{(n-1) / 2} K_{e}[\dot{\varepsilon}]^{(p-1) / 2}
$$

where $\mu, \dot{\gamma}$ and $\dot{\varepsilon}$ are the viscosity, shear rate and strain rate, respectively, and $K_{v}, K_{e}, p$ and $n$ are (known) material properties. It is to be noted that, $K_{v}, K_{e}, p$ and $n$ are material properties specific to each polymer solution. In practice, they must be determined by curve-fitting to viscometric data obtained in shear and extensional flows. It is also worthmentioning that the power-law exponent $n$ controls the degree of shear-thinning of a fluid. Similarly, the power-law exponent $p$ controls the degree of a fluid's strain-thickening behavior; it can also be regarded as a measure of a fluid's elasticity. Polymer solutions are known to be shear-thinning $(n<1)$ and strain-thickening $(p>1)$. In contrast, for Newtonian fluids we have $n=p=1$. The simple viscosity model as given by Eq. 1 can account for both shear-thinning and strainthickening behavior of polymeric liquids. This viscosity model is applicable to laminar and turbulent flows as long as the flow is one-dimensional. For fully-developed steady turbulent pipe flows, the viscosity can be decomposed into a time-averaged mean value, $\bar{\mu}$, plus a viscosity fluctuation, $\mu^{\prime}$. That is, at any given location within the pipe, one can write $\mu(t)=\bar{\mu}+\mu^{\prime}(t)$. Pinho ${ }^{21)}$ derived the following closedform expression for the time-averaged (mean) molecular viscosity:

$$
\begin{aligned}
\bar{\mu} & =\left(C_{\mu} \rho\right)^{3 m(m-1) A_{2} /\left(8+3 m(m-1) A_{2}\right)} \\
& \times 2^{4 m(m-1) A_{2} /\left(8+3 m(m-1) A_{2}\right)} k^{6 m(m-1) A_{2} /\left(8+3 m(m-1) A_{2}\right)} \\
& \times B^{8 /\left(8+3 m(m-1) A_{2}\right)}
\end{aligned}
$$

where $k$ and $\varepsilon$ are turbulent kinetic energy and its rate of dissipation, respectively. In this equation, $C_{\mu}$ is a standard parameter in $k-\varepsilon$ models with a value which depends on the turbulence model selected for the analysis (to be introduced shortly), $A_{2}$ is a constant equal to 0.45 which is deemed to be universal, at least, for Newtonian fluids ${ }^{21)}$, and $m$ and $B$ are related to the rheology and flow kinematics as ${ }^{21)}$ :

$$
\left\{\begin{array}{l}
m=\frac{n+p-2}{n+p} \\
B=\left[\frac{K_{v} K_{e}}{A_{\varepsilon}{ }^{P-1}}\right]^{1-m} 2^{[(n-1)-m(n+1)] / 2} \rho^{m}
\end{array}\right.
$$

with $A_{\varepsilon}$ being a parameter which quantifies the ratio between the fluctuating strain and shear rates. ${ }^{23)}$ From a parametric investigation onto its effects upon the model predictions, Cruz and Pinho ${ }^{22)}$ assigned a numerical value of 10 to $A_{\varepsilon}$. As will be shown shortly, $A_{\varepsilon}$ should preferably be determined within an inverse scheme to better match experimental data for dilute polymer solutions.

Equation 2 is known to be good for high Reynolds-number turbulence. In regions very close to the wall, however, molecular viscosity should reduce to pure shear viscosity. That is, no extensional effect is conceivable near the wall because there is virtually no fluctuation near in this region with the flow being essentially unidirectional in this region. To take this into account, Cruz and Pinho ${ }^{22)}$ introduced the following damping function for the molecular viscosity, $f_{v}$ :

$$
\bar{\mu}=f_{v} \bar{\mu}_{h}+\left(1-f_{v}\right) \eta_{v}
$$

Assuming that this damping function is the same as the damping function for the eddy viscosity, Cruz and Pinho ${ }^{22)}$ derived the following form for $f_{v}$ which takes into account both shear and extensional properties of the fluid:

$$
\begin{aligned}
& f_{v}=\left\{1-\left[1+\left|\frac{1-n}{1+n}\right| y^{+}\right]^{-|(1+n) /(1-n)| / A^{+}}\right\} \\
& \times\left\{1-\left[1+\left|\frac{p-1}{3-p}\right| y^{+} C^{(1-p) /(2-p)}\right]^{-|(3-p) /(p-1)| / A^{+}}\right.
\end{aligned}
$$

where $A^{+}$is the so-called Van Driest universal constant equal to 26.5. It is to be noted that, $y^{+}$is the wall coordinate defined by $y^{+}=u_{\tau} y / \bar{v}_{w}$, where $u_{\tau}$ is the friction velocity and $\bar{v}_{w}$ is the kinematic viscosity right at the wall. The parameter $C$ appearing in this equation is an adjusting parameter which can be used for fitting numerical predictions to the experimental data. Cruz and Pinho ${ }^{22)}$, and Cruz et al. ${ }^{23)}$ stressed that the correct strategy for evaluating this parameter is through an inverse scheme. In practice, however, they relied on a trial-and-error method for its determination. That is, using rheological data for a $0.125 \%$ Polyacrylamide (PAA) polymer solution, several values of $C$ were tried and the predictions of $f$-Re were compared with the experimental data. Using this approach, $C$ was determined to be equal to 70 . This value for $C$ was then used to obtain numerical results for other polymer solutions. $^{26,27)}$ But $C=70$ is not consistent with the order-ofmagnitude analysis developed by Pinho for turbulent flows of GNFs. ${ }^{21)}$ As discussed in Ref. ${ }^{21)}$, for the order of magnitude analysis to be valid $\mathrm{C}$ should always remain of order one. As will be shown shortly, this parameter can be optimized through an inverse scheme such that it remains (nearly) of order one. 
Having decided on the constitutive equation of the fluid, the next step is to insert it in the equations of motion. For statistically-stationary, fully-developed turbulent pipe flow of any fluid, the x-momentum equation is the only equation that needs to be considered. In cylindrical coordinates, this equation can be written as:

$$
0=\frac{1}{r} \frac{d}{d r}\left[r\left(\bar{\mu} \frac{d U}{d r}-\rho \overline{u v}+2 \overline{\mu^{\prime} s_{x y}}\right)\right]-\frac{d \bar{p}}{d x}
$$

where $x$ is the flow direction, $r$ is the radial direction, $U$ is the average velocity, $\bar{p}$ is the mean pressure, $\mu^{\prime}$ is the viscosity fluctuation, and $-\rho \overline{u v}$ is the Reynolds shear stress. The last term inside the parenthesis (namely, $2 \overline{\mu^{\prime} s_{x y}}$ ) is a new stress term resulting from a correlation which exists between fluctuations in the molecular viscosity and fluctuations in the velocity-gradient. This new stress term has been modeled by Cruz et $a .^{23)}$ as:

$$
\begin{aligned}
2 \overline{\mu^{\prime} s_{x y}} & =\frac{\widetilde{C}}{L_{c}} \frac{K_{v} K_{e}}{A_{\varepsilon}{ }^{p-1}}\left[\frac{\rho C_{\mu} f_{\mu} k^{2}}{2 \bar{\mu} \widetilde{\varepsilon}}\left(\frac{d U}{d r}\right)^{2}\right]^{(p+n-2) / 2} \\
& \times \sqrt{C_{\mu} f_{\mu} \frac{k^{2}}{\widetilde{\varepsilon}}} \frac{d U / d r}{\sqrt{|d U / d r|}}
\end{aligned}
$$

where we have:

$$
\widetilde{C}=\left(1+C_{0}\right)^{p+n-2}-1
$$

Due to lack of sufficient experimental data, Cruz et al. ${ }^{23)}$ used this ratio as an adjusting parameter which was determined through a trial-and-error method to be equal to 3 . In the present work, $C_{0}$, too, will be determined through an inverse method. In Eq. 7, $L_{c}$ represents spatial scale of the turbulence eddies and is defined $b y^{25)}$ :

$$
\left\{\begin{array}{l}
\frac{1}{L_{c}}=\frac{\varepsilon}{u_{R}^{3}} \\
u_{R}=\sqrt{\frac{k}{\left|\exp \left[-\left(k / u_{\tau}{ }^{2}\right)^{4}\right]-1\right|^{1 / 4}}}
\end{array}\right.
$$

with $u_{\tau}$ being the friction velocity. Now we are at a stage to invoke a suitable low-Reynolds number turbulence model to our fluid mechanics problem. But the use of such low-Re models requires some modifications to be made to the main transport equations for $k$ and $\varepsilon$. The first modification is a redefinition of the rate of dissipation $(\tilde{\varepsilon})$ which is needed to facilitate implementation of wall boundary conditions. The true rate of dissipation, $\varepsilon$, is related to the modified rate, $\tilde{\varepsilon}$, by the following relationship:

$$
\varepsilon=\widetilde{\varepsilon}+D
$$

where $D$ is dependent on the low-Reynolds number turbulence model selected for the simulations (to be introduced shortly). Using the modified rate-of-dissipation function $(\tilde{\varepsilon})$, Cruz et $a .^{23)}$ modified the transport equations for $k$ and $\varepsilon$ as:

$$
\begin{aligned}
0= & \frac{1}{r} \frac{d}{d r}\left[r\left(\bar{\mu}+\frac{\rho v_{T}}{\sigma_{k}}\right) \frac{d k}{d r}\right]-2 \overline{\mu^{\prime} s_{x y}} \frac{d U}{d r} \\
& -\rho \overline{u v} \frac{d U}{d r}-\rho \widetilde{\varepsilon}-\rho D \\
0= & \frac{1}{r} \frac{d}{d r}\left[r\left(\bar{\mu}+\frac{\rho v_{T}}{\sigma_{\varepsilon}}\right) \frac{d \widetilde{\varepsilon}}{d r}\right]+\rho f_{1} C_{\varepsilon 1} \frac{\widetilde{\varepsilon}}{k} P \\
& -\rho f_{2} C_{\varepsilon 2} \frac{\widetilde{\varepsilon}^{2}}{k}+\rho E+C_{\varepsilon 4} \frac{v_{T}}{\sigma_{\varepsilon} \bar{v}} \frac{d \widetilde{\varepsilon}}{d r} \frac{d \bar{\mu}}{d r}
\end{aligned}
$$

where $P$ is the turbulence production term, $v_{T}$ is the eddy viscosity, $\sigma_{k}$ is the turbulent Prandtl number for $k$ equation, and $\sigma_{\varepsilon}$ is the turbulent Prandtl number for the $\varepsilon$ equation. In this equation, $E, f_{1}, f_{2}, C_{\varepsilon 1}$ and $C_{\varepsilon 2}$ all depend on the lowReynolds turbulence model selected for the analysis (to be introduced shortly). The last term in the rate of dissipation equation, Eq. 12, takes into account the effect of spatial variation of the molecular viscosity, and so requires some knowledge about the constitutive behavior of the fluid. This term includes a new coefficient, $C_{\varepsilon 4}$, which was set equal to one by Cruz and Pinhor ${ }^{22)}$, and Cruz et al. ${ }^{23)}$ because of the lack of sufficient experimental data. In the present work, we have decided to consider this coefficient as an unknown parameter and determine its numerical value via the inverse scheme. In above equations, the Reynolds shear stress is modeled as:

$$
-\rho \overline{u v}=\rho v_{T} \frac{d U}{d r}
$$

where $U$ is the average velocity, and $v_{T}$ is the eddy viscosity defined by:

$$
v_{T}=C_{\mu} f_{\mu} \frac{k^{2}}{\widetilde{\varepsilon}}
$$

The formulations presented above contain several parameters (namely, $D, E, f_{1}, f_{2}$ ) which depend on the selection of the low-Reynolds number $k-\varepsilon$ model adopted for the analysis. The turbulence model used by Cruz and Pinho ${ }^{22)}$, and Cruz et $a{ }^{23)}$ for their numerical simulations was the NaganoHishida model ${ }^{28)}$ For this particular turbulence model these parameters are: 


$$
\begin{aligned}
& D=2 \bar{v}\left(\frac{d \sqrt{k}}{d r}\right)^{2} \\
& E=\bar{v} v_{T}\left(1-f_{\mu}\right)\left(\frac{d^{2} U}{d r^{2}}\right)^{2} \\
& f_{1}=1.0 ; f_{2}=1-0.3 \exp \left(-R e_{T}{ }^{2}\right)
\end{aligned}
$$

where the turbulent Reynolds number is defined as $R e_{T}=\frac{k^{2}}{v \widetilde{\varepsilon}}$. The remaining parameters of this low-Re turbulence model (which are all based on correlation with Newtonian data because of the lack of sufficient data for non-Newtonian fluids) have been listed in Table I.

To find out the optimized values for the parameters mentioned above (i.e., $C, C_{0}, C_{\varepsilon 4}, A_{\varepsilon}$ ) using inverse methods, we are going to rely on the rheological data for $0.125 \%$ PAA solution (which is also the sample selected randomly in previous studies for the numerical calibrations ${ }^{22,23)}$ ). Table II presents rheological parameters of this particular polymer solution along with rheological data for several other polymer solutions (to be used later on for further model checking).

\section{THE INVERSE PROBLEM}

As mentioned above, Cruz and Pinho ${ }^{22)}$ assigned numerical values to the parameters of interest (i.e., $C, C_{0}, C_{\varepsilon 4}, A_{\varepsilon}$ ) either through a trial-and-error approach. In the inverse method developed in the present work, these parameters will be optimized in a systematic way to better match experimental data. Fortunately, for our test fluid (i.e., $0.125 \%$ PAA solution) experimental data are available for friction factor, velocity profile, and turbulent kinetic energy. This problem, in essence, is a multi objective problem. This multi objective

Table I. Parameters of the Nagano-Hishida low-Reynolds number $k-\varepsilon$ model.

\begin{tabular}{ccccc}
\hline$C_{\mu}$ & $\sigma_{k}$ & $\sigma_{\varepsilon}$ & $\sigma_{\varepsilon 1}$ & $\sigma_{\varepsilon 2}$ \\
\hline 0.09 & 1.0 & 1.3 & 1.45 & 1.9 \\
\hline
\end{tabular}

Table II. Test samples and their rheological properties. ${ }^{26,27)}$

\begin{tabular}{lcccc}
\hline Solution & $K_{v}$ & $n$ & $K_{e}$ & $p$ \\
\hline $0.125 \%$ & 0.24 & 0.42 & 8.25 & 1.47 \\
PAA & 91 & 50 & 00 & 96 \\
\hline \multirow{2}{*}{$0.2 \%$ PAA } & 0.78 & 0.40 & 9.86 & 1.31 \\
& 49 & 75 & 50 & 75 \\
\hline \multirow{2}{*}{$0.2 \%$ XG } & 0.27 & 0.44 & 3.85 & 1.25 \\
& 01 & 09 & 19 & 92 \\
\hline
\end{tabular}

problem is treated by combining the multiple objectives into one scalar objective, whose solution is a Pareto optimal point for the original Multi Objective Problem (MOP). ${ }^{28)}$ A standard technique for MOP is to minimize a positively weighted convex sum of the objectives. This technique is applicable to nonlinear problems. The objective function is constructed by a sum of squared differences between experimental values and numerical predictions for each of the three dependent variables mentioned above. To be more specific, our objective function is:

$$
\begin{aligned}
S= & w_{1} \sum_{i=1}^{m_{1}}\left(Y_{i}^{1}-T_{i}^{1}(\beta)\right)^{2}+w_{2} \sum_{j=1}^{m_{2}}\left(Y_{j}^{2}-T_{j}^{2}(\beta)\right)^{2} \\
& +w_{3} \sum_{k=1}^{m_{3}}\left(Y_{k}^{3}-T_{k}^{3}(\beta)\right)^{2}
\end{aligned}
$$

where $Y^{1}, Y^{2}$ and $Y^{3}$ are experimental data for friction factor, velocity profile and turbulent kinetic energy, and $T^{1}, T^{2}$ and $T^{3}$ are their corresponding predicted numerical values with $w_{1}, w_{2}, w_{3}$ serving as the weighting functions. In Eq. $18, m_{1}$ is the number of friction factors (measured at different Reynolds numbers), and $m_{2}$ and $m_{3}$ represent different positions across the pipe cross-section.

In order to minimize the objective function as given by Eq. 18, the multi-objective version of the well-known GaussNewton $(G N)$ non-linear optimization method will be used (see Appendix for more details). The $G N$ method is an iterative scheme rendering optimized values for the unknown parameters $\left(C, C_{0}, C_{\varepsilon 4}, A_{\varepsilon}\right)$ through minimizing the function $S$.

\section{COMPUTATIONAL PROCEDURE}

The computational procedure for estimating the unknown parameters at each iteration can be summarized as follows (see Ref. ${ }^{3)}$ for more details):

1. Make an initial guess for all parameters, $P^{i}$.

2. Solve the direct problem with these tentative values for the pertinent parameters in order to calculate quantities of interest (e.g., velocity profile, friction factor, etc).

3. Compute $S$ from Eq. 18.

4. Compute the sensitivity matrixes $X^{i}$ defined by:

$$
X^{i}=\left[\begin{array}{cccc}
\frac{\partial T_{1}^{i}}{\partial P_{1}} & \frac{\partial T_{1}^{i}}{\partial P_{2}} & \cdots & \frac{\partial T_{1}^{i}}{\partial P_{n}} \\
\frac{\partial T_{2}^{i}}{\partial P_{1}} & \frac{\partial T_{2}^{i}}{\partial P_{2}} & \cdots & \frac{\partial T_{2}^{i}}{\partial P_{n}} \\
\cdot & \cdot & \cdots & \cdot \\
\frac{\partial T_{m}^{i}}{\partial P_{1}} & \frac{\partial T_{m}^{i}}{\partial P_{2}} & \cdots & \frac{\partial T_{m}^{i}}{\partial P_{n}}
\end{array}\right]
$$


where $i=1,2,3$, and $T$ represents each dependent variable.

5. Solve the following linear system of equations in order to find $\Delta P$.

$$
\begin{aligned}
& {\left[w_{1} \cdot\left(X^{1}\right)^{T(i)}\left(X^{1}\right)^{(i)}+w_{2} \cdot\left(X^{2}\right)^{T(i)}\left(X^{2}\right)^{(i)}\right.} \\
& \left.+w_{3} \cdot\left(X^{3}\right)^{T(i)}\left(X^{3}\right)^{(i)}\right] \Delta P^{(i)} \\
& =\left(X^{1}\right)^{T} \cdot\left[Y^{1}-T^{1}\right]+\left(X^{2}\right)^{T} \cdot\left[Y^{2}-T^{2}\right] \\
& \quad+\left(X^{3}\right)^{T} \cdot\left[Y^{3}-T^{3}\right]
\end{aligned}
$$

6. Update the guessed parameter (to be used for the next iteration) as:

$$
P^{(i+1)}=P^{(i)}+\Delta P^{(i)}
$$

7. Solve the direct problem again this time using the updated values for the parameters, $P^{i+1}$, in order to calculate $T\left(P^{i+1}\right)$.

8. Calculate $S$ using the updated $T\left(P^{i+1}\right)$ and stop the whole process if the convergence criterion (say, $S \leq \lambda$ with $\lambda$ being a small number) is met; otherwise, repeat steps 2 to 8 : In practice, Oliveira and Orlande ${ }^{30)}$, and Colaco and Orlande ${ }^{31)}$ suggested the following relationship for $\lambda$ :

$$
\lambda=N \Gamma^{2}
$$

where $N$ is the number of measurements, and $\Gamma$ is the standard deviation for the experimental data. It is to be noted that, since experimental data normally contain random errors, therefore, we cannot expect the function $S$ to become exactly equal to zero no matter how many iterations we choose.

\section{RESULTS AND DISCUSSION}

Based on the methodology described above, the numerical simulations were carried out with a finite volume code developed by Mehrabadi ${ }^{32)}$ and run on a Pentium-4 PC to find out the best values for all parameters of interest (namely, $C$, $\left.C_{0}, C_{\varepsilon 4}, A_{\varepsilon}\right)$. The code was first validated by comparing its output for Newtonian fluids with known results for this kind of fluids. ${ }^{31)}$ It was then used to calculate the above parameters for the fluid of interest (that is, $0.125 \%$ PAA solution). To initiate our inverse analysis for determining the abovementioned parameters, all these parameters were set equal to one as the initial guess. The weighting functions $\left(\mathrm{w}_{1}, \mathrm{w}_{2}, \mathrm{w}_{3}\right)$ as appearing in Eq. 20 were realized to affect the numerical results. To decide on the best values for these weighting functions, use could have been made of the direct assignment method, the eigenvector method, the entropy method, and the minimal information method, among others. ${ }^{33,34)}$ In the present work, we have decided to rely on the direct assignment method for determining the weighting functions with the constraint that they should satisfy the relationship $\sum_{i=1}^{3} w_{i}=1$. That is to say that, we resorted to a trial-and-error method in order to obtain the best values for the weighting functions. The numerical value adopted for each of the three weighting functions $\mathrm{w}_{1}, \mathrm{w}_{2}, \mathrm{w}_{3}$ (obtained using the trial-and-error method) was equal to $1 / 3$.

Of particular importance in the inverse analysis is having a good knowledge about the degree of correlation between the unknown parameters themselves. To that end, the condition number of the sensitivity matrix should also be determined with great care. These two issues directly affect the stability and thus convergence of the gradient-based estimation process. Computation of the condition number (say, using the Fisher information matrix method) has the further advantage that it can allow the assessment of any ill-conditioned characteristics of the estimation problem. ${ }^{35,36)}$ The condition number of the Fisher information matrix, $X^{T} X$, can be defined as

$$
\text { cond. }=\frac{\left|\lambda_{1}\right|}{\left|\lambda_{n p}\right|}
$$

where $\lambda_{1}$ and $\lambda_{n p}$ are the largest and smallest eigenvalues of the $X^{T} X$ matrix, respectively, and $n p$ is the rank of this matrix. Having constructed the Fisher information matrix, the condition number was determined to be equal to $7.93 \times 10^{9}$. Such a huge condition number means a large difference between the highest and the lowest sensitivity coefficients, and so a linear dependence between the parameter sensitivity coefficients. The correlation matrix for the parameters of interest, determined using the procedure described by Walpole and Myers $^{35)}$, have been tabulated in Table III.

The strong correlation between $C$ and $A_{\varepsilon}$, and also between $C_{0}$ and $A_{\varepsilon}$ means that simultaneous determination of these two sets of parameters is not an easy task. Of prime importance in the optimization process is deciding on the number of iterations after which the calculations can be stopped. Lacking the information needed about the error inherent in the experimental data, it was decided to plot reduction history of the objective function for the friction factor, velocity profile

Table III. Correlation matrix between the parameters.

\begin{tabular}{ccccc}
\hline Parameter & $C$ & $C_{0}$, & $C_{\varepsilon 4}$ & $A_{\varepsilon}$ \\
\hline$C$ & 1.0000 & -0.8691 & 0.6598 & -0.9376 \\
\hline$C_{0}$, & -0.8691 & 1.0000 & -0.6624 & 0.9738 \\
\hline$C_{\varepsilon 4}$ & 0.6598 & -0.6624 & 1.0000 & -0.5981 \\
\hline$A_{\varepsilon}$ & -0.9376 & 0.9738 & -0.5981 & 1.0000 \\
\hline
\end{tabular}


and turbulent kinetic energy with respect to the number of iterations, $n$. Figures (1-a) to (1-c) present such graphs all suggesting that the total number of iterations required to reach to a given accuracy is between 20 to 30 . In practice, however, the code was let to run even above these numbers until a sudden drop occurred in the calculated error at which point the inverse computation was deemed to have converged to its desired point.

Table IV presents the optimized values for the parameters $C$, $C_{0}, C_{\varepsilon 4}, A_{\varepsilon}$ calculated using the inverse method just described. For comparison purposes, Table IV also includes the values adopted by Cruz et $a .^{23)}$ for the same parameters. The difference between the output of the inverse method and those adopted by Pinho appear to be significant, particularly for $A_{\varepsilon}$. Of particular importance is the notion that the inverse method gives a value of roughly 18 for $C$. This should be contrasted with a numerical value of 70 as used in $\operatorname{Ref}^{23)}$ for $C$. Since the order-of-magnitude analysis used for formulating turbulent flow problem of GNFs relies on $C$ being small, it is evident that the inverse method used in the present work is providing a better value for $C$.

At this stage we would like to stress that our inverse method has revealed that amongst different model parameters, the three parameters most affected by the inverse method are $\mathrm{C}$, $C_{0}$, and $A_{\varepsilon}$. We have already discussed the importance of $\mathrm{C}$ and the more suitability of the one obtained in this work using the inverse method (i.e., $C=18$ vs. $C=70$ as used in Ref. ${ }^{23)}$ ). The inverse method also shows that $C_{0}$ should be increased from 3 to roughly 80 . The parameter $C_{0}$ represents the ratio of averaged deformation rate to the r.m.s. of the fluctuations in the deformation rate. Thus any increase in $C_{0}$ will increase the magnitude of the new stress terms. In practice, however, its effect was found to be minimal and so could safely be neglected. In contrast, any change in $A_{\varepsilon}$ affects the magnitude of the new stress termr ${ }^{23)}$ quite dramatically with a subsequent effect on the quality of the final results. In fact, the optimized value for $A_{\varepsilon}$ as found in this work suppresses the magnitude of this new stress term to roughly 0.1 of its previous value. Since the new stress term is negative ${ }^{23)}$, it plays the same role as the production term in the $k$-equation, and so leads to a smaller turbulent kinetic energy production, as can be observed in Figure 4. As a result, the eddy viscosity is also dropped suggesting that the turbulent diffusion should drop to some extent. Finally we would like to add that the remaining parameter optimized in this work, i.e., $C_{\varepsilon 4}$, is quite close to that offered by Cruz et al. ${ }^{23)}$ through trial-and-error method.

To check the significance of the optimized values listed in Table IV, they were used to calculate the friction factor, velocity profile, and turbulent kinetic energy in wall coordinates for the $0.125 \%$ PAA polymer solution in turbulent pipe flow. The non-uniform mesh used in the computer code developed in this work had 100 cells from the wall to the center-line for an axisymmetric situation. The code relies

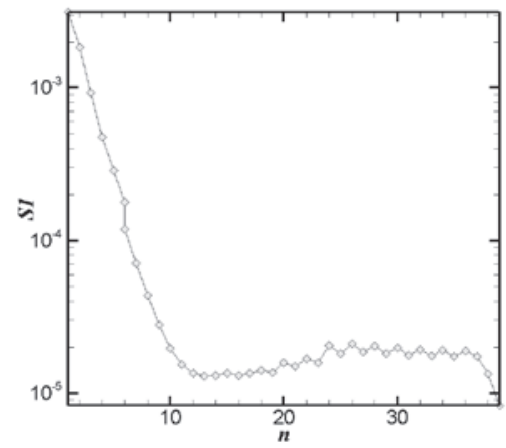

(1-a)

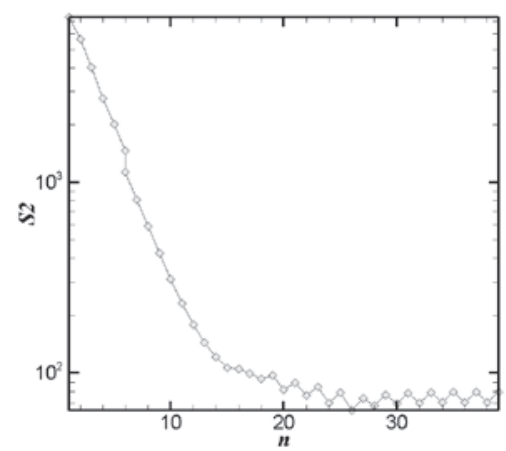

$(1-b)$

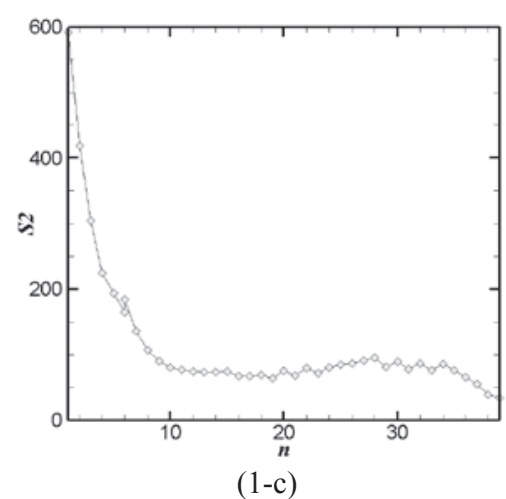

Fig. 1. Reduction history of the objective function for:(a) friction factor, (b) velocity profile, and (c) turbulent kinetic energy.

Table IV. A comparison between parameters obtained using inverse method with those reported in. ${ }^{26,27)}$

\begin{tabular}{ccc}
\hline Parameter & $\begin{array}{c}\text { Inverse Method } \\
\text { (present work) }\end{array}$ & $\begin{array}{c}\text { Direct Method } \\
\text { Ref. }^{22}\end{array}$ \\
\hline$C$ & 18 & 70 \\
\hline$C_{0}$ & 79 & 3 \\
\hline$C_{\varepsilon 4}$ & 0.75 & 1 \\
\hline$A_{\varepsilon}$ & 1298 & 10 \\
\hline
\end{tabular}


on more than at least 15 control volumes in the viscous sublayer. This particular mesh was selected for the simulations after preliminary studies showed that it gives rise to meshindependent results within $0.1 \%$.

Figure 2 shows numerical results for the friction factor calculated using the optimized parameters obtained in the present work from our inverse method. The Reynolds number is defined as:

$$
\operatorname{Re}=\frac{U_{b} D}{\bar{v}_{w}}
$$

where $U_{b}$ is the bulk velocity and $\bar{v}_{w}$ is the fluid's viscosity at the wall. This figure also includes a reproduction of the results obtained using the parameters suggested by Cruz and Pinho $^{22)}$ with the code developed in. ${ }^{31)}$ As seen in this figure, our optimized parameters are doing a better job in predicting the friction factor. ${ }^{23)}$ Figure 3 compares the performance of the optimized "parameters" with those used by Cruz et al. ${ }^{23)}$ in relation to the dimensionless velocity profile, defined by $u^{+}=u / u_{\tau}$. As seen in this figure, close to the wall (i.e., in the viscous sublayer) the performance of both sets of

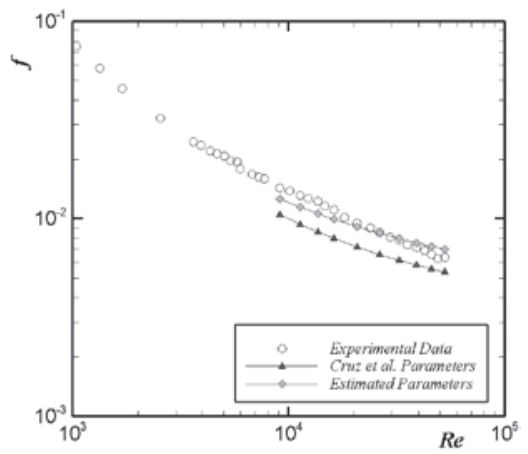

Fig. 2. A comparison between numerical results for the friction factor, $f$, obtained using our optimized parameters with those obtained by Cruz et al. ${ }^{23)}$ for a $0.125 \%$ PAA solution.

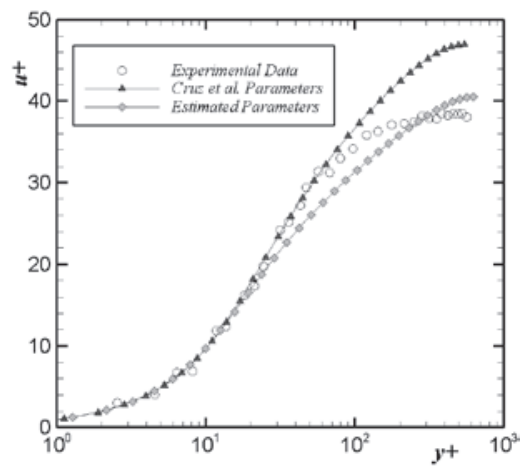

Fig. 3. A comparison between numerical results for the velocity profile, $u^{+}$, obtained using our optimized parameters with those obtained by Cruz et al. ${ }^{23)}$ for a $0.125 \%$ PAA solution. parameters is virtually the same. In the overlap layer, in fact, the performance of the old values appears to be better, but, in the outer layer it is the optimized values which evidently are doing a better job.

Figure 4 compares the performance of new values for the "parameters" with those used by Cruz et al. ${ }^{23)}$ for nondimensional turbulent kinetic energy, namely $k^{+}=k / u_{\tau}^{2}$, in wall coordinates. Close to the wall, the predictions of both new and old values for the parameters are virtually the same. Outside the viscous sublayer, however, the performance of the optimized parameters appears to be much better than the old ones.

Figure 5 presents a comparison between the performance of the old and new parameters in predicting $f$-Re curve for a $0.2 \%$ PAA polymer solution, that is, another concentration of the same polymer PAA. Although, the performance of the optimized parameters appear to be slightly better, but what is more striking is the notion that both sets of results are different from experimental data quantitatively (qualitatively, however, they predict the trend correctly). This suggests that the parameters obtained in this work, and also those used by Cruz et al. ${ }^{23)}$, are concentration-dependent.

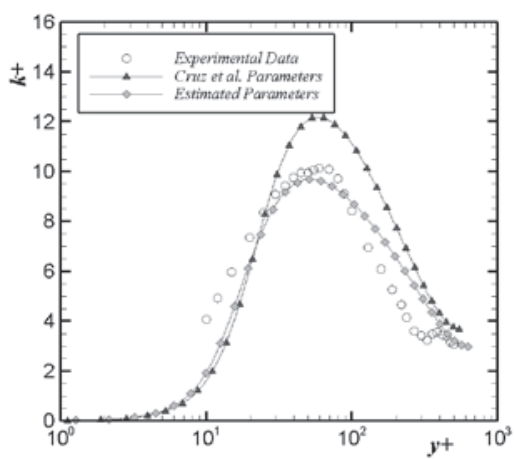

Fig. 4. A comparison between numerical results for the kinetic energy, $\mathrm{k}+$, obtained using our optimized parameters with those obtained by Cruz et al. ${ }^{23)}$ for a $0.125 \%$ PAA solution.

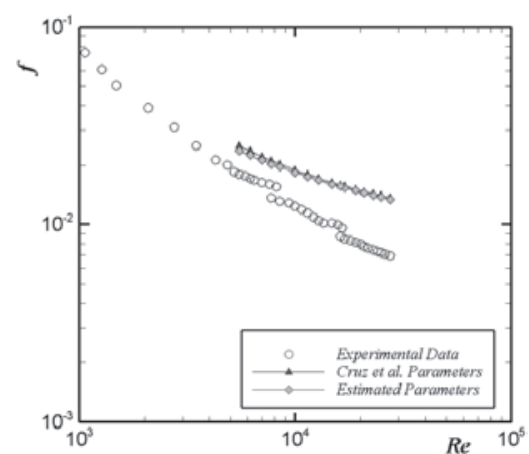

Fig. 5. A comparison between numerical results for the friction factor, $f$, obtained using our optimized parameters with those obtained by Cruz et al. ${ }^{23)}$ for a $0.2 \%$ PAA solution. 
That is to say that, even for a given polymer these parameters should be used with cautious. Figures 6 to 8 show numerical predictions for the friction factor, velocity profile, and turbulence kinetic energy for $0.2 \%$ Xanthan Gum (XG) polymer solution using both the optimized parameters and the old parameters. Although, in general, optimized parameters appear to be slightly superior to those used by Cruz et $a .^{23)}$, quantitatively, however, agreement with experiment is not that great. This finding has the implication that even these optimized parameters are not universal but polymerdependent.

\section{CONCLUSIONS}

The present work has demonstrated quite clearly the importance of the numerical values which must be to certain parameters in turbulent flow modeling of dilute polymer solution in circular Poiseuille flow. These parameters, which appear in the rate of dissipation function, the eddy viscosity damping function, and also the apparent polymeric stress, has been determined using a trial and error approach by

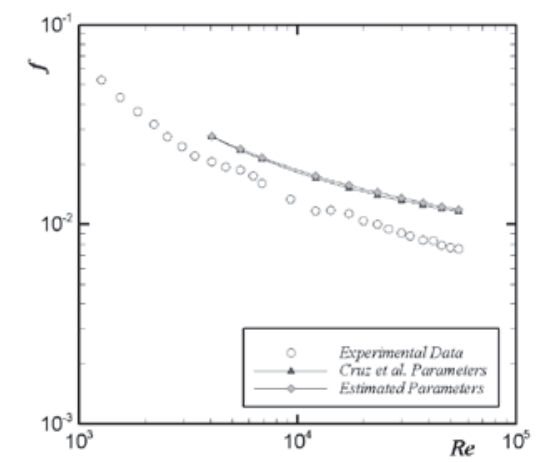

Fig. 6. A comparison between numerical results for the friction factor, $f$, obtained using our optimized parameters with those obtained by Cruz et al. ${ }^{23)}$ for a $0.2 \% X G$ solution.

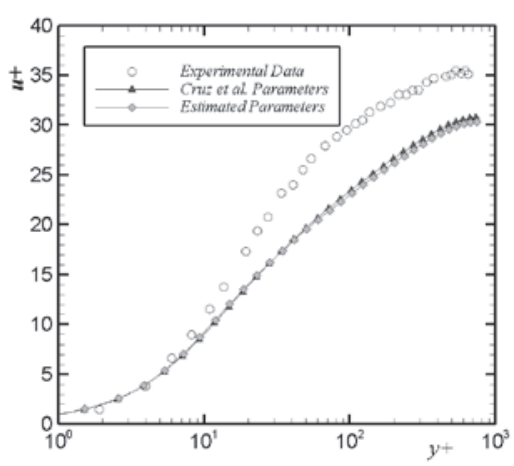

Fig. 7. A comparison between numerical results for the velocity profile, $u^{+}$, obtained using our optimized parameters with those obtained by Cruz et $a l .^{23)}$ for a $0.2 \% X G$ solution.
Cruz et al.. ${ }^{22,23)}$ It is shown that better numerical prediction can be made for the friction factor if these parameters are optimized using a multi-objective Gauss-Newton optimization method. The present work also suggests that the parameters optimized using experimental data for a given polymer solution at a given concentration may not work for other concentrations of even the same polymer, let aside other polymers. Indeed, it was observed in our investigations that for XG polymer, numerical simulation for the friction factor may be in significant error if parameters pertinent to the PAA polymer are used. The results reported in the present work suggests that in order to improve the quality of numerical results in predicting the drag-reducing effect of any polymeric additive, it might be a good idea to resort to inverse methods for deciding on the numerical values of more parameters appearing in the formulations, not just the four parameters optimized in this work.

\section{Acknowledgement}

The authors would like to thank research council of the University of Tehran for supporting this work. Thanks are also due to the reviewers for their constructive comments.

\section{APPENDIX}

An extension of the Gauss method was developed in the present work to minimize a multi-objective function. The objective functional for this problem can be written as:

$$
\begin{aligned}
& S=w_{1} \sum_{i=1}^{m_{1}}\left(Y_{i}^{1}-T_{i}^{1}(\beta)\right)^{2}+w_{2} \sum_{j=1}^{m_{2}}\left(Y_{j}^{2}-T_{j}^{2}(\beta)\right)^{2} \\
& +w_{3} \sum_{k=1}^{m_{3}}\left(Y_{k}^{3}-T_{k}^{3}(\beta)\right)^{2}
\end{aligned}
$$

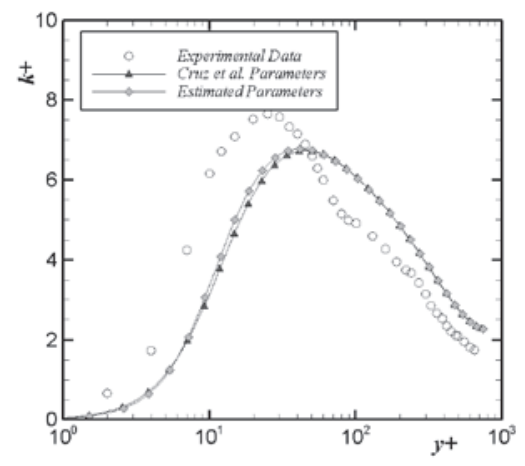

Fig. 8. A comparison between numerical results for the kinetic energy, $k^{+}$, obtained using our optimized parameters with those obtained by Cruz et al. ${ }^{23)}$ for a $0.2 \% X G$ solution. 
In matrix form, the objective function can be written as:

$$
\begin{aligned}
S= & w_{1}\left[Y^{1}-T^{1}(\beta)\right]^{T}\left[Y^{1}-T^{1}(\beta)\right] \\
& +w_{2}\left[Y^{2}-T^{2}(\beta)\right]^{T}\left[Y^{2}-T^{2}(\beta)\right] \\
& +w_{3}\left[Y^{3}-T^{3}(\beta)\right]^{T}\left[Y^{3}-T^{3}(\beta)\right]
\end{aligned}
$$

The (matrix) derivative of the objective function with respect to $\beta$ is:

$$
\begin{aligned}
\nabla_{\beta} S & =2 w_{1}\left[\nabla_{\beta}\left(T^{1}\right)^{T}(\beta)\right]\left[Y^{1}-T^{1}(\beta)\right] \\
& +2 w_{2}\left[\nabla_{\beta}\left(T^{2}\right)^{T}(\beta)\right]\left[Y^{2}-T^{2}(\beta)\right] \\
& +2 w_{3}\left[\nabla_{\beta}\left(T^{3}\right)^{T}(\beta)\right]\left[Y^{3}-T^{3}(\beta)\right]
\end{aligned}
$$

The minimization process requires this equation to be set to zero at $\beta=\hat{\beta}$. To that end we substitute:

$$
\left(X^{i}(\beta)\right)^{T}=\left[\nabla_{\beta}\left(T^{i}\right)^{T}(\beta)\right] \quad ; \quad i=1,2,3
$$

By setting Eq. A3 equal to zero, one would obtain:

$$
\begin{aligned}
& w_{1}\left(X^{1}(\hat{\beta})\right)^{T}\left[Y^{1}-T^{1}(\hat{\beta})\right] \\
& \quad+w_{2}\left(X^{2}(\hat{\beta})\right)^{T}\left[Y^{2}-T^{2}(\hat{\beta})\right] \\
& \quad+w_{3}\left(X^{3}(\hat{\beta})\right)^{T}\left[Y^{3}-T^{3}(\hat{\beta})\right]=0
\end{aligned}
$$

At this stage, we assume that we have an estimation of $\hat{B}$ at hand denoted by $b$. Thus we can replace $X(\hat{B})$ by $X(b)$ We can also expand $T(\hat{\beta})$ around $b$ in a Taylor's series. Keeping only the first two terms of this series, we will obtain:

$$
\begin{aligned}
& w_{1}\left(X^{1}(b)\right)^{T}\left[Y^{1}-T^{1}(b)-\left[\nabla_{\beta}\left(T^{1}\right)^{T}(b)\right]^{T}(\hat{\beta}-b)\right] \\
& +w_{2}\left(X^{2}(b)\right)^{T}\left[Y^{2}-T^{2}(b)-\left[\nabla_{\beta}\left(T^{2}\right)^{T}(b)\right]^{T}(\hat{\beta}-b)\right] \\
& +w_{3}\left(X^{3}(b)\right)^{T}\left[Y^{3}-T^{3}(b)-\left[\nabla_{\beta}\left(T^{3}\right)^{T}(b)\right]^{T}(\hat{\beta}-b)\right]=0
\end{aligned}
$$

The above equation can be recast in the following form using our notation for the sensitivity (see Eq. 19):

$$
\begin{aligned}
& w_{1}\left(X^{1}(b)\right)^{T}\left[Y^{1}-T^{1}(b)-\left(X^{1}(b)\right)(\hat{\beta}-b)\right] \\
& +w_{2}\left(X^{2}(b)\right)^{T}\left[Y^{2}-T^{2}(b)-\left(X^{2}(b)\right)(\hat{\beta}-b)\right] \\
& +w_{3}\left(X^{3}(b)\right)^{T}\left[Y^{3}-T^{3}(b)-\left(X^{3}(b)\right)(\hat{\beta}-b)\right]=0
\end{aligned}
$$

We now substitute:

$$
\begin{aligned}
& b^{(k)}=b, b^{(k+1)}=\hat{\beta} \\
& T^{i(k)}=T^{i}(b),\left(X^{i(k)}\right)=\left(X^{i}(b)\right)
\end{aligned}
$$

Using these new variables, Eq. A7 becomes:

$$
\begin{aligned}
b^{(k+1)} & =b^{(k)}+P^{(k)}\left(X^{1(k)}\right)^{T}\left[w_{1}\left[Y^{1}-T^{1(k)}\right]\right. \\
& +\left(X^{2(k)}\right)^{T} w_{2}\left[Y^{2}-T^{2(k)}\right] \\
& \left.+\left(X^{3(k)}\right)^{T} w_{3}\left[Y^{3}-T^{3(k)}\right]\right]
\end{aligned}
$$

bwhere:

$$
\begin{array}{r}
P^{-1(k)} \equiv\left[\left(X^{1(k)}\right)^{T} w_{1}\left(X^{1(k)}\right)\right. \\
+\left(X^{2(k)}\right)^{T} w_{2}\left(X^{2(k)}\right) \\
\left.+\left(X^{3(k)}\right)^{T} w_{3}\left(X^{3(k)}\right)\right]
\end{array}
$$

This way, a new set of the unknown parameters $b^{(k+1)}$ is obtained. Since Eq. A8 has been obtained by keeping the first two terms of the Taylor's series only, an iterative procedure is needed for finding the unknown parameters of a nonlinear problem.

\section{REFERENCES}

1) Hensel E, "Inverse Theory and Applications for Engineers. Prentice Hall, Englewood Cliffs", (1991) New Jersey.

2) Beck JV, Blackwell B, Clair Jr, "Inverse Heat Conduction: IllPosed Problems", (1985) John Wiley, New York.

3) Beck JV, Arnold KJ, "Parameter Estimation in Engineering and Science", (1977) John Wiley, New York.

4) Tikhonov AN, Arsenin VY. "Solution of Ill-Posed Problem", (1977) Winston and Sons, Washington, DC.

5) Alifanov OM, “Inverse Heat Transfer Problems”, (1994) Springer-Verlag, New York.

6) Levenberg K, Q Appl Math, 2, 164 (1944).

7) Marquardt DW, J Soc Ind Appl Math, 11, 431 (1963).

8) Ozisik MN, Orlande HRB, "Inverse Heat Transfer: Fundamentals and Applications”, (2000) Taylor \& Francis, New York.

9) Pourshaghaghy A, Kowsary F, Behbahaninia A, Heat and Mass Transfer, 3, 285 (2007).

10) Goldberg DE, "Genetic Algorithms in Search, Optimization and Machine Learning”, (1989) Addison Wesley, Reading, MA.

11) Garcia S, Scott EP, Num Heat Transfer A, 33, 135 (1998).

12) Garcia S, Guynn J, Scott EP, Num Heat Transfer, Part A, 33, 149 (1998).

13) Liu FB, Ozisik MN, Int J Heat Mass Transfer, 39, 2615 (1996).

14) Li HY, Yan WM, Int J Heat Mass Transfer, 46, 1041 (2003).

15) Su J, Lopes AB, Silva Neto AJ, Int Commun Heat Mass Transfer, 27, 945 (2000).

16) Su J, Silva Neto AJ, Num Heat Transfer A, 40, 751 (2001). 
17) Brasil WM, Su J, Freire APS, Int J Heat Mass Transfer, 47, 1267 (2004).

18) Storcha RB, Pimentela LCG, Orlande HRB, Atmospheric Environment, 41, 1417 (2007).

19) King AC, Bloor MIG, The Quarterly J of Mech and App Mathematics, 42,2, 183 (1989).

20) Toms BA, Proceedings of the First International Congress on Rheology, Amsterdam, 2, 135 (1948).

21) Pinho FT, J of Non-Newtonian Fluid Mech, 114, 149 (2003).

22) Cruz DOA, Pinho FT, J Non-Newtonian Fluid Mech, 114, 109 (2003).

23) Cruz DOA, Pinho FT, Resende PR, J Non-Newtonian Fluid Mech, 121, 127 (2004).

24) Resende PR, Escudier MP, Presti F, Pinho FT, Cruz DOA, Int. J. of Heat and Fluid Flow, 27, 204 (2006).

25) Allahdadi-Mehrabadi MA, Sadeghy K, Mech Research Comm, accepted for publication (2008).

26) Escudier MP, Presti F, Smith S, J Non-Newtonian Fluid Mech, 81, 197 (1999).

27) Presti F, "Investigation of transitional and turbulent pipe flow of non-Newtonian fluids", (2000) Ph.D. Thesis, University of Liverpool, UK.
28) Nagano Y, Hishida M, J Fluids Eng, 156 (1987).

29) Lin JG, “Three Methods for Determining Pareto-Optimal Solutions of Multiple-Objective Problems. Directions in LargeScale Systems", (1975) Plenum Press, New York.

30) Oliveira APD, Orlande HRB, Inverse Problems in Science and Engineering, 5, 563 (2004).

31) Colaco MJ, Orlande HRB, Numerical Heat Transfer A, 36, 229 (1999).

32) Allahdadi-Mehrabadi M, “Application of k- $\varepsilon$ turbulence model for predicting drag reduction in poisuillie flow with the use of polymer additives", (2006) M.Sc Thesis, University of Tehran, Iran.

33) Abraham A, Jain L, Goldberg R, "Evolutionary Multiobjective Optimization Theoretical Advances and Applications”, (2005) Springer-Verlag, London Limited.

34) Tan KC, Khor EF, Lee TH, "Multiobjective Evolutionary Algorithms and Applications”, (2005) Springer-Verlag, London Limited.

35) Kiefer J, Biometrika, 62, 2, 277 (1975).

36) Walpole RE, Myers RH, "Probability and Statistics for Engineers and Scientists”, 5th Ed, (1993) Macmillan Publishing Co, New York. 